\title{
Mechanical behaviour of a creased thin strip
}

\author{
Jie Liu ${ }^{1,2}$, Shanqing $\mathrm{Xu}^{1}$, Guilin $\mathrm{Wen}^{2}$, and Yi Min $\mathrm{Xie}^{1,3}$ \\ ${ }^{1}$ Centre for Innovative Structures and Materials, School of Engineering, \\ RMIT University, Melbourne 3001, Australia \\ ${ }^{2}$ State Key Laboratory of Advanced Design and Manufacturing for Vehicle Body, \\ Hunan University, Changsha 410082, China \\ ${ }^{3}$ XIE Archi-Structure Design (Shanghai) Co., Ltd., Shanghai 200092, China \\ Correspondence: Yi Min Xie (mike.xie@rmit.edu.au)
}

\begin{abstract}
Received: 20 October 2017 - Revised: 22 December 2017 - Accepted: 30 January 2018 - Published: 20 February 2018
Abstract. In this study the mechanical behaviour of a creased thin strip under opposite-sense bending was investigated. It was found that a simple crease, which led to the increase of the second moment of area, could significantly alter the overall mechanical behaviour of a thin strip, for example the peak moment could be increased by 100 times. The crease was treated as a cylindrical segment of a small radius. Parametric studies demonstrated that the geometry of the strip could strongly influence its flexural behaviour. We showed that the uniform thickness and the radius of the creased segment had the greatest and the least influence on the mechanical behaviour, respectively. We further revealed that material properties could dramatically affect the overall mechanical behaviour of the creased strip by gradually changing the material from being linear elastic to elasticperfect plastic. After the formation of the fold, the moment of the two ends of the strip differed considerably when the elasto-plastic materials were used, especially for materials with smaller tangent modulus in the plastic range. The deformation patterns of the thin strips from the finite element simulations were verified by physical models made of thin metal strips. The findings from this study provide useful information for designing origami structures for engineering applications using creased thin strips.
\end{abstract}

Origami has been gaining increasing interest in recent years due to its extraordinary features and potential applications (Chen et al., 2015; Filipov et al., 2015; Dias et al., 2012). The fundamental principle of origami is to transform a flat sheet square (2-D) of paper into a finished sculpture (3-D) through folding and sculpting techniques along pre-defined creases. Because of its unique properties, origami has been imitated and developed to design foldable mechanisms (Hanna et al., 2014), self-deployable structures (Delimont et al., 2015), robots (Jayaram and Full, 2016), self-folding structures (Na et al., 2015), metamaterials (Overbelde et al., 2017), energy absorbing structures (Yang et al., 2016, 2017) and to solve plant structure folding (Couturier et al., 2013), soft matter folding (Lin et al., 2016) and even protein folding problems (Gethin and Sambrook, 1992). From an engineering viewpoint, mechanical properties of the crease for design- ing origami structures are of significant importance, which should be fully understood and characterised. Unfortunately, the knowledge of the contribution of the introduced creases to the overall mechanical behaviour is still limited.

Generally, the crease connecting two adjacent rigid facets was treated as an elastic hinge with specific stiffness (Yasuda and Yang, 2015). Yasuda and Yang (2015) investigated the bistable nature of the re-entrant 3-D origami structure. They modelled the structure by connecting the rigid plates with torsional spring along the creased lines. However, the deformation of the plates might have a considerate influence on the mechanical response of the origami structure (Lechenault et al., 2014). Schenk and Guest (2011) treated the folding pattern as a pin-jointed framework. The stiffness of origami structure in their work was derived by using a stiffness matrix approach. Their work simplified the origami structure as discrete trusses instead of continuous one. In addition, they only assumed that the materials of trusses were linear elastic. 
Dias and Audoly (2014) employed a singular elastic hinge line to model the crease, which did not necessarily need to be straight. Francis et al. (2013) defined a hinge parameter to assess the crease of non-paper sheet materials. Their research could guide designers to material selection and origami designs.

Due to the significant influence of creases on the origami structure, the mechanical properties of the creases should be characterised based on their geometry and material properties, instead of assumptions. Lechenault et al. (2014) experimentally and analytically investigated the mechanics of a creased thin Mylar sheet. They introduced a characteristic length scale, aiming to find a generic feature that could govern the mechanism of structural response when a small load was applied, which might consist in angle opening or panel bending. Walker and Seffen (2016) studied the mechanical behaviour of a thin metallic creased strip. They folded the creased strip along its axis by equal-sense bending (the direction of folding was the same as the opening of the origami structure). The crease was regarded as a cylindrical segment of a small radius and its real material properties were considered. However, very little research could be found to study the mechanical response of the creased thin strip folded by opposite-sense bending (the fold direction was opposite to the opening direction of the origami structure), which is of practical value for possibly higher bending stiffness. In opposite-sense bending of the creased thin strip, the crease would lead to the increase of the second moment of area, which could significantly influence its overall mechanical behaviour, thus, designers should concern the role of the creases specifically in opposite-sense bending.

The equal-sense bending and opposite-sense bending of tape springs have been extensively studied and broadly applied to self-deployable structures in aerospace field, which could offer help in investigating the opposite-sense bending of the creased strips. Seffen and Pellegrino (1999) studied the dynamic deployment of a tape spring that is used as a deployment actuator. They found that the process of emerging and growth of elastic folds in tape springs is a propagating instability, which could be represented by a high peak moment and a smaller propagation moment. In their study, they mainly focused on the straight tape spring. Later, Seffen et al. (2000) investigated the bending process of the curved tape springs, and they demonstrated that the mechanical behaviours of the straight tape spring and the curved tape spring were almost in common except for equal-sense folds with small rotation angles. For more studies on the tape spring, the readers are referred to Soykasap (2007), Walker and Aglietti (2006), Yang et al. (2015), Ye et al. (2017) and references therein.

In this paper, we attempted to identify the mechanical behaviour of a creased thin strip by opposite-sense bending. Inspired by Walker and Seffen's work, we treated the crease as a cylindrical segment of a small radius. We first tried to verify our finite element modellings for the creased thin strip

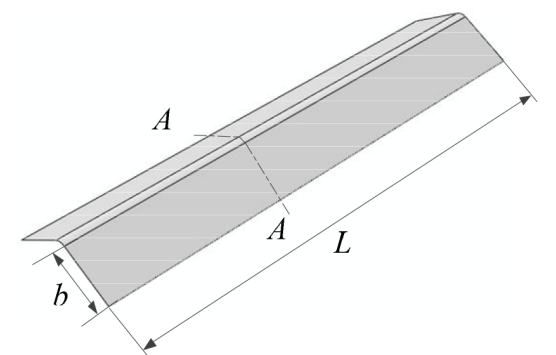

Section $A-A$

(a)

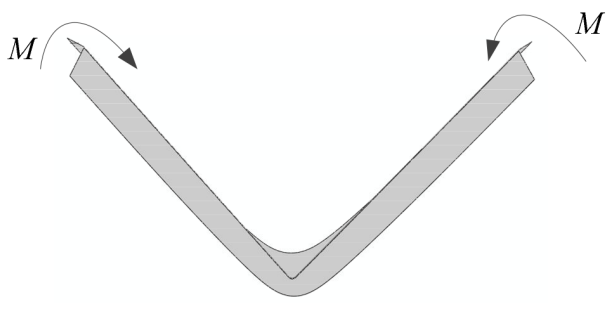

(b)

Figure 1. (a) The geometry of an undeformed creased strip and its cross-section and (b) localized fold at the middle of the creased strip by opposite-sense bending.

by comparing the finite element analyse (FEA) results and the experiment results adopted from Steffen et al. (2000) for the opposite-sense bending of a curved strip. Based on this, we studied the mechanical response of bending a creased thin strip. The influence of the geometry on the overall mechanical response was investigated. We also systematically studied the transition from a creased strip to a curved strip. Furthermore, the influence of on the mechanical behaviour of the creased strip was investigated.

\section{Opposite-sense bending of creased thin strips}

Opposite-sense bending of creased thin strips was studied in this paper. The crease region of the strip was treated as a cylindrical segment of a small radius, as shown in Fig. 1a. Thus the strip was simplified as the combination of two rectangular facets of the same size and a curved crease. The key dimensions of the strip were the radius of crease curvature $(r)$, the length of the strip $(L)$, the width of the rectangular facet $(b)$, the uniform thickness of the strip $(t)$, and the initial opening angle $(\theta)$.

The creased strip was bent in opposite sense, as shown in Fig. 1b. Assuming that there was no external axial, shear or torsional force exerted on the strip, the bending of the strip could be treated as a pure bending problem. Note that in Fig. 1b, $M$ represented the moment applied at the end of the strip, which was generated by rotational displacements at the two ends of the strip. 

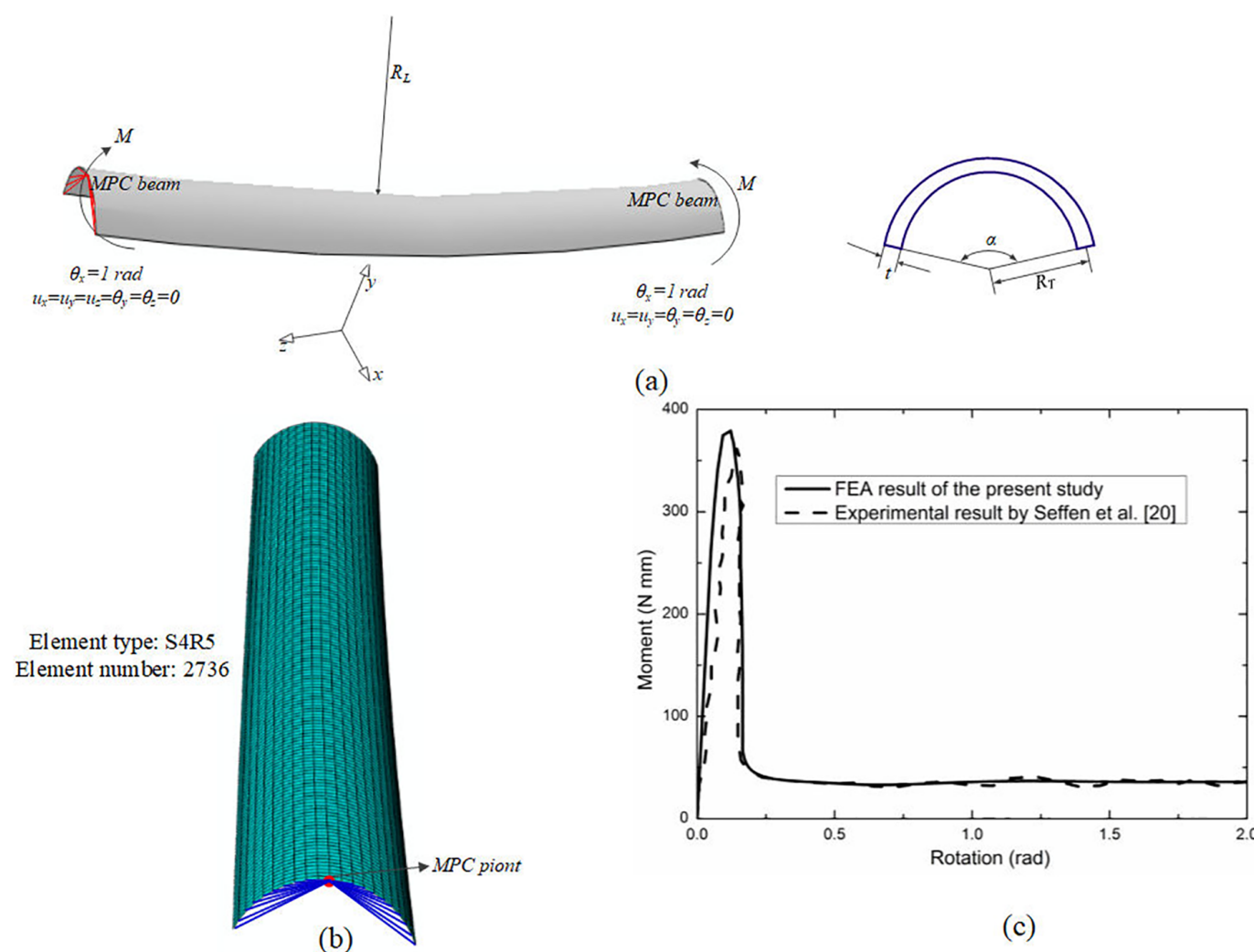

(c)

Figure 2. (a) Curved strip with negative Gaussian curvature and subject to a moment couple $M>0$ at both ends and the boundary condition (left), and the cross-section of the strip (right), (b) the mesh and the boundary conditions of the strip, and (c) the moment-rotation relationship obtained from the finite element modelling of curved strip as compared to that by Seffen et al. (2000).

\section{Finite element analyses}

\subsection{Verification of the finite element model}

The experimental result by Seffen et al. (2000) on the mechanical response of bending a curved strip was employed in this paper to verify the finite element model. A curved strip adapted from Steffen et al. (2000) was analyzed (Fig. 2a), with dimensions and material properties listed in Table 1 . In the model, the strip direction was set to be the $z$ axis Cartesian coordinate and the $y$ axis was vertically perpendicular to the $z$ axis. The displacement components of the strip ends were denoted as $u_{x}, u_{y}$, and $u_{z}$, respectively. The rotational displacement components of the strip were denoted as $\beta_{x}$, $\beta_{y}$, and $\beta_{z}$, respectively. The boundary condition for applying a moment was defined by $u_{x}=u_{y}=u_{z}=\beta_{y}=\beta_{z}=0$ and $\beta_{x}=1$ radian for the left end of the strip, and $u_{x}=u_{y}=$ $\beta_{y}=\beta_{z}=0$ and $\beta_{x}=-1$ radian for the other end. Therefore, the degree-of-freedom in $z$ direction for the right end of the strip was released, which led to the asymmetrical boundary conditions. It should be noted that the curved strip in the work of Seffen et al. (2000) was initially doubly curved but this work focused on initially straight creased strips. In the finite element modelling by Abaqus standard, the rota- tional displacement was implemented at the end of the strip The shell was meshed by using 4-node reduced integration shell elements with five degrees of freedom per node (S4R5), making up 2736 elements (see Fig. 2b). Multiple point constraints (MPC) were used to define the boundary conditions. The nodes at both ends of the strip were tied to an MPC node on the same plane at the middle of the arc by rigid beam elements to ensure their synergetic movements, thus achieving pure bending, as shown in Fig. 2b. Dissipated energy fraction was used to enable the analysis to converge. The analysis method in Abaqus was static by considering geometric large deformation. The material was assumed to be linear elastic.

The relationship between the moment and the rotational angle was plotted in Fig. 2c and compared with the experimental result by Seffen et al. (2000). It showed good agreement between our result and theirs. Therefore, all the finite element analyses of this paper were conducted based on the verified model. It should be mentioned that, although Seffen et al. (2000) indicated that their experiment results were valid only for decreasing rotation amplitudes, it could also valid for increasing rotation amplitudes if a linear elastic material was used. 


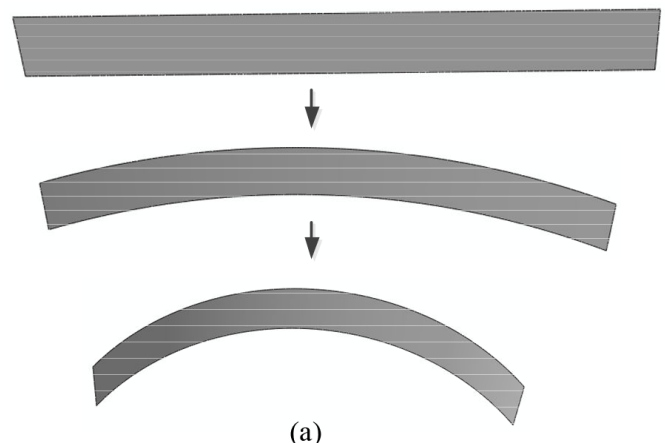

(a)

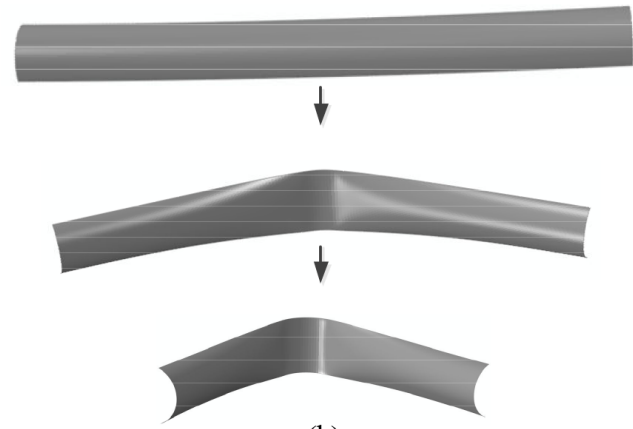

(b)

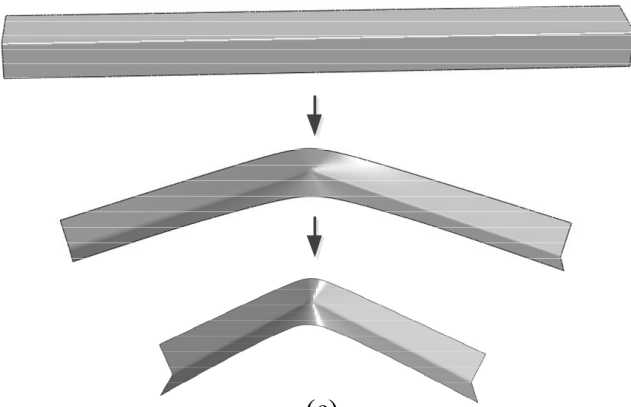

(c)

Figure 3. Folding processes by finite element simulations for (a) flat strip, (b) curved strip, and (c) creased strip.

Table 1. Material properties and geometrical dimensions of the curved strip for FEA verification.

\begin{tabular}{lr}
\hline Designation & Value \\
\hline Young's modulus $E$ & $131000 \mathrm{~N} \mathrm{~mm}^{-2}$ \\
Poisson's ratio $v$ & 0.3 \\
Thickness $t$ & $0.1 \mathrm{~mm}$ \\
Length $L$ & $220 \mathrm{~mm}$ \\
Subtended angle $\alpha$ & $2.39 \mathrm{rad}$ \\
Transverse radius $R_{\mathrm{T}}$ & $11.5 \mathrm{~mm}$ \\
Longitudinal radius $R_{\mathrm{L}}$ & $1380 \mathrm{~mm}$ \\
\hline
\end{tabular}

\subsection{FEA of folding creased thin strips}

For the convenience of investigating purely the structural influence, the material for making the strips was kept unchanged in the FEA. Furthermore, to make sure that all the strips could be made of one same thin flat strip, their crosssectional shapes shall be kept identical. In the FEA, at least four elements were used across the crease to assure the simulation accuracy and quality. Assuming that all the strips had the same length and thickness, and the radius of the strip, $R_{\mathrm{L}}$, was sufficiently large (the strips were approximately initially straight), a geometrical constraint could be identified to define the relationship among the width of the rectangular facets $(b)$, the radius of the crease segment $(r)$ and the initial opening angle $(\theta)$

$(\pi-\theta) r+2 b=\alpha R_{\mathrm{T}}$ where $R_{\mathrm{T}}$ was the transverse radius of the curved strip, $\alpha$ was the subtended angle of the curved strip. In this way, different types of strips could be made by using the same flat thin plate. For example, a set of geometrical parameters for creating a creased strip with a fixed cross-sectional area could be identified according to Eq. (1), namely, $b=13.70 \mathrm{~mm}$, $r=0.1 \mathrm{~mm}$, and $\theta=2.09 \mathrm{rad}$, which will be used in the following finite element analyse to compare the mechanical behaviour with those of flat and curved strips. For the flat strip, its cross-sectional area, thickness and length were the same with those of the curved and the creased strips. It should be noted that the values of $R_{\mathrm{L}}$ were infinite for the creased and flat straight strips, where $R_{\mathrm{L}}$ was the longitudinal radius.

The bending process of three types of strips, i.e., flat strip, curved strip and creased strip, were numerically analyzed by using the verified finite element model as shown in Fig. 3. In order to qualitatively verify the final deformed shapes of strips in the finite element modelling, physical models of the same arrangement were tested in bending and the deformed shapes of the three types of strips were shown in Fig. 4. It should be emphasised that only the final deformed shapes of strips were verified and linear-elastic materials similar to Steffen et al. (2000) were applied with the non-linear deformation being neglected in the studied bending range, in which the influence of non-linear deformation was insignificant. In the experiments, flat, curved and folded thin metal strips were fixed at the two ends by using mixed polyamide resin and ethoxyline resin. All the thin metal strips had the same length $(200 \mathrm{~mm})$ and thickness (roughly 


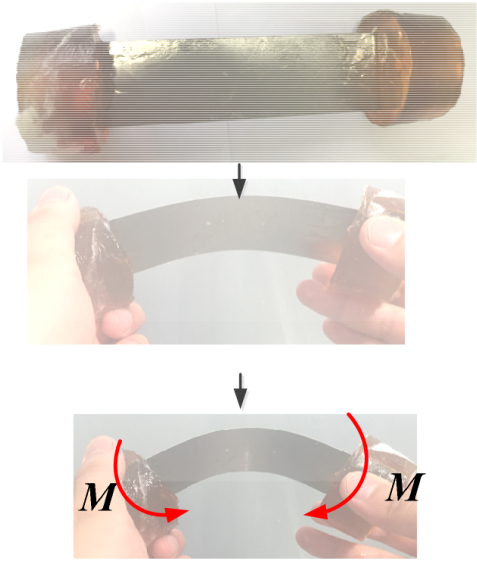

(a)

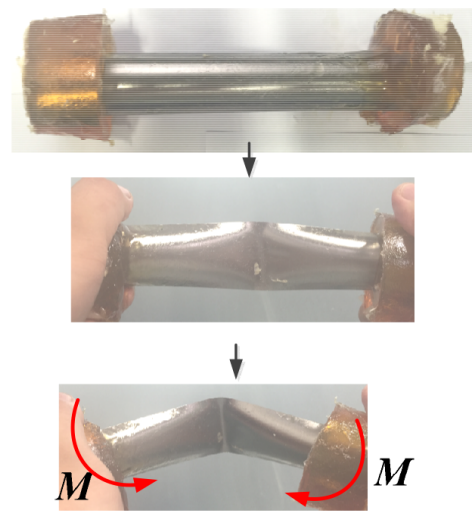

(b)

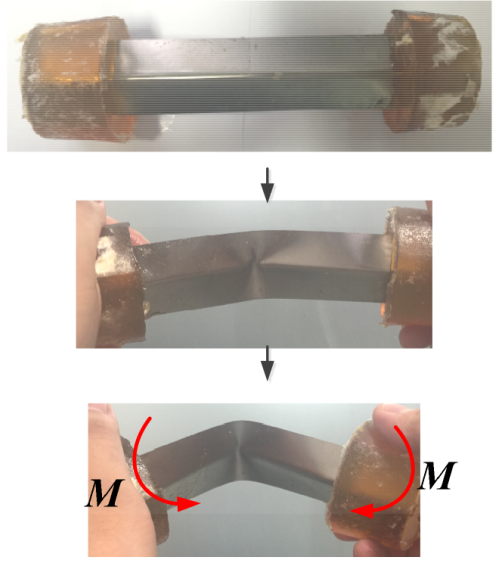

(c)

Figure 4. Folding processes of physical models for (a) flat strip, (b) curved strip, and (c) creased strip.

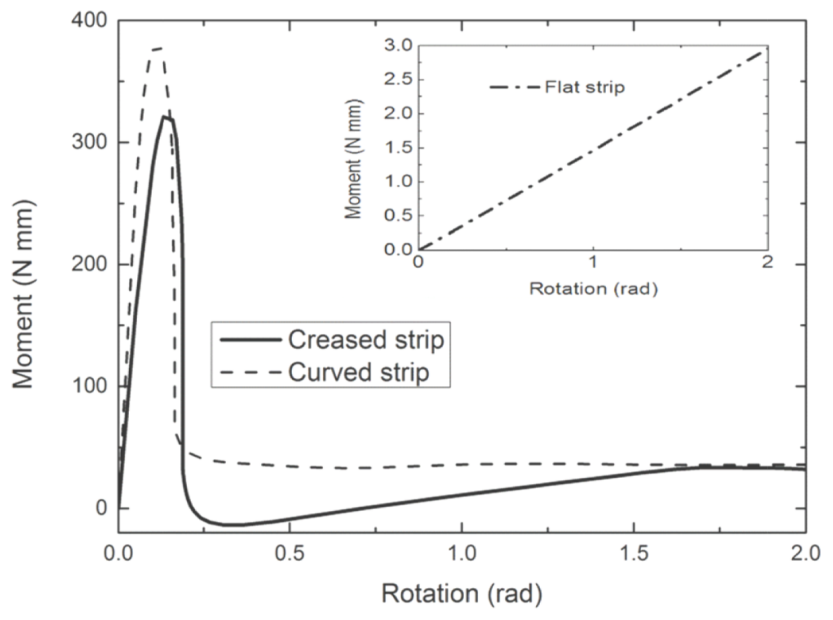

Figure 5. Moment-rotation relationship obtained from finite element modelling showing the comparison for the flat strip, the curved strip, and the creased strip.
$0.3 \mathrm{~mm}$ ). It should be noted that we have prepared and tested six specimens by using the vernier caliper, and the maximum/minimum value for the thickness is $0.27 \mathrm{~mm} / 0.31 \mathrm{~mm}$. The opening angle for the creased strip was approximately $2.09 \mathrm{rad}$. We bended the flat, curved and folded thin metal strips using two hands, respectively. Each hand held the mixed polyamide resin and ethoxyline resin. We used the camera to capture three typical statuses during the bending process for each strip. Specifically, the first status is the original state, namely, before bending; the second status is when $\beta_{x}=0.5$ radian; the third status is when $\beta_{x}=1$ radian. The experiments showed good agreement between experiments and numerical simulations in terms of the deformation. The deformed shape of the flat strip was smooth and continuous without localized fold (see Figs. 3a and 4a). Localized folds existed at the middle of both the creased strip and the curved strip, but in different patterns. For creased strip, the fold was localized in a very small region adjacent to the crease line, seemingly a small folding line (see Figs. $3 \mathrm{c}$ and $4 \mathrm{c}$ ). While for curved strip the localized fold occupied a fairly larger region with smooth transition (see Figs. $3 b$ and $4 b$ ). 


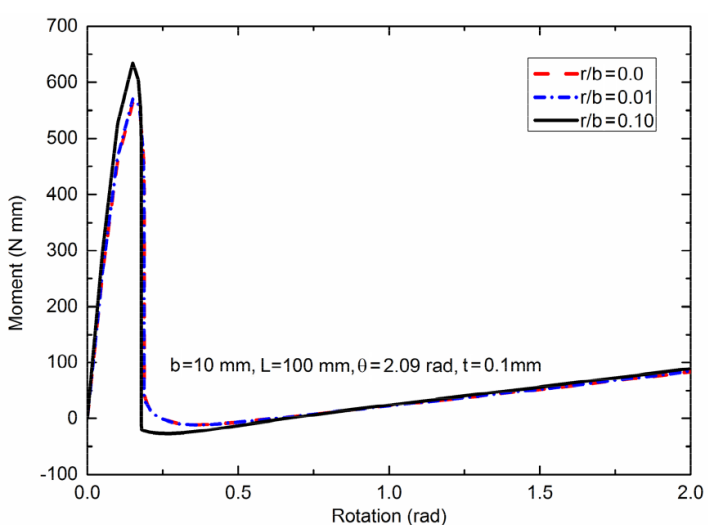

(a)

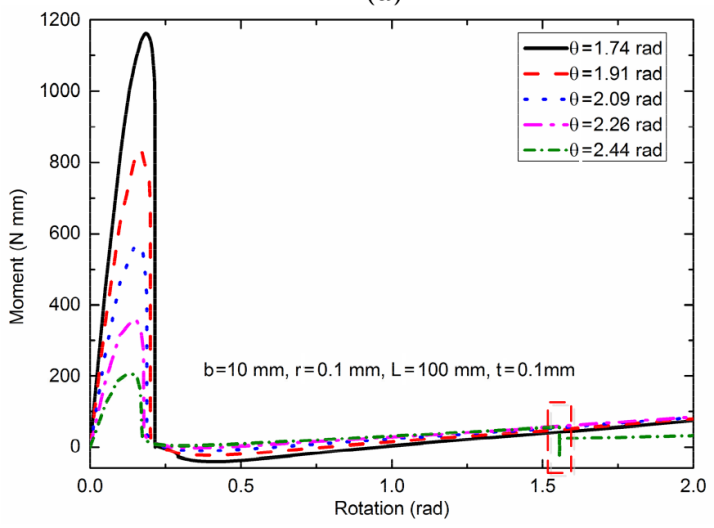

(c)

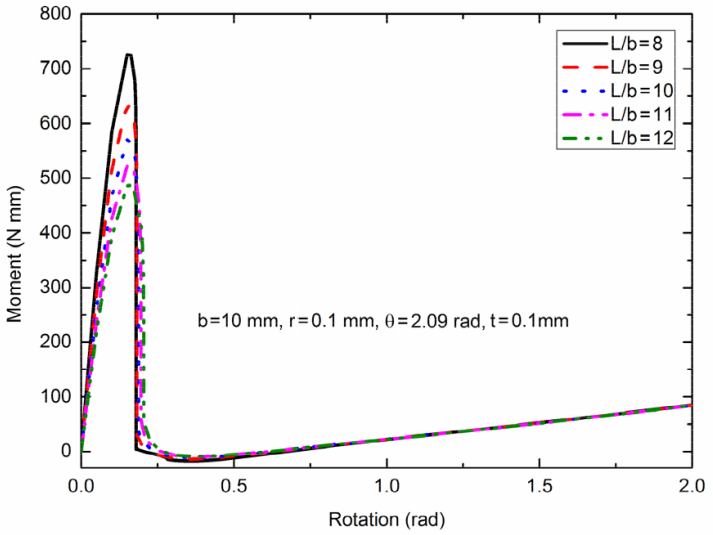

(b)

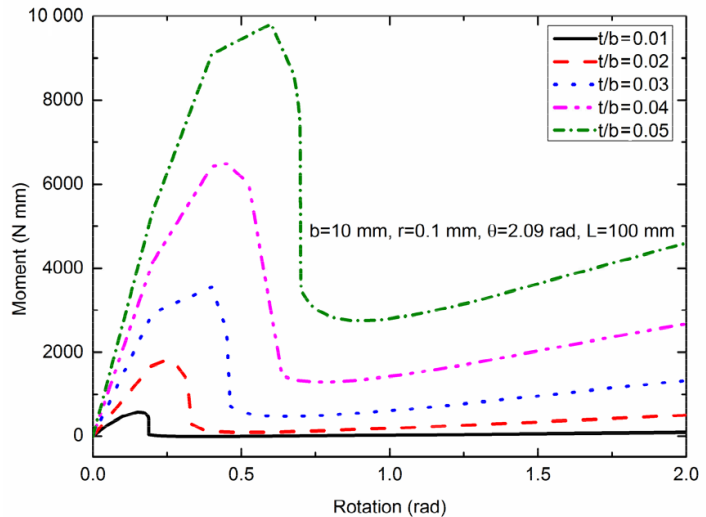

(d)

Figure 6. Moment-rotation relationship obtained from finite element modellings showing the influences of nondimensional geometrical parameters of the strips on the mechanical responses: (a) $r / b$, (b) $L / b$, (c) $\theta$, and (d) $t / b$.

Figure 5 compared the moment-rotation curves of the three strips from the finite element simulations. In the bending process, flat strip showed a linear relationship between the moment and the rotational angle with negligible moment in the studying range as compared to the other two strips. The moment of both the creased and curved strips rocketed up after a very small rotation in the initial stage, peaking at values above $300 \mathrm{~N} \mathrm{~mm}$. With the occurrence of localized folds, the moment dropped instantly to much smaller values of below $50 \mathrm{~N} \mathrm{~mm}$. Interestingly, at this stage, the moment of the curved strip would maintain at around $50 \mathrm{Nmm}$. While the moment of creased strip would suddenly drop to a negative moment value and steadily increase with the proceeding of rotation, then plateau at around $50 \mathrm{~N} \mathrm{~mm}$. The sudden drop of the moment was attributed to the formation of localized folds. Unlike the curved strip, an even serious "snapthrough" occurred during the bending of the creased strip. In the studied case, the creased strip had a smaller peak bending moment than the curved one. Actually, the peak bending moment was highly associated with the geometrical parameters of the strip. Thus, a detailed parametric study on creased strips was conducted and analyzed in the following section.

\section{Parametric studies}

Various finite element simulations were conducted to study the influence of the dimensionless geometrical parameters on the mechanical response of the creased strip, including radius of crease curvature to width ratio $(r / b)$, strip length to width ratio $(L / b)$, initial opening angle $(\theta)$, and strip thickness to width ratio $(t / b)$. The value of the width of the rectangular facet $(b)$ was fixed at $10 \mathrm{~mm}$ for all the analyses. The transition from a creased strip to a curved strip was also systematically investigated.

\subsection{Radius of crease curvature to width ratio, $r$ / $b$}

To investigate the influence of $r / b$ on the mechanical response of the creased strip, the length of the strip $(L)$, the initial opening angle $(\theta)$, and the uniform thickness of the strip $(t)$ were fixed at $100 \mathrm{~mm}, 2.09 \mathrm{rad}, 0.1 \mathrm{~mm}$, respectively, i.e., $L=100 \mathrm{~mm}, \theta=2.09 \mathrm{rad}, t=0.1 \mathrm{~mm}$. The radius of crease curvature $(r)$ varied from 0 to $1 \mathrm{~mm}$ (small $r / b$ the values) so that the length of the curvature is sufficiently smaller than the width of facet of the origami fold. When $r / b=0$, there was no curvature for the crease line, meaning the two facets 
(a)

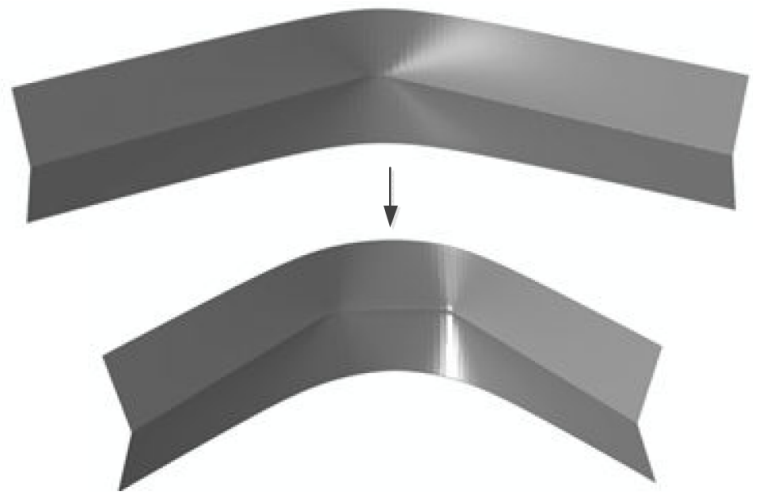

Figure 7. The transformation of localized folding from (a) single fold to (b) double folds.

were jointed directly. Figure 6a illustrated the mechanical responses of creased strips of various $r / b$ values under bending. Typical shapes as described previously were observed for all the strips. With the increase of $r / b$, the peak moment increased. Furthermore, there was negligible difference in the moment when $r / b$ was smaller than 0.01. Further increase of $r / b$ above 0.01 resulted in seemingly larger increment of the moment. It should be pointed out that with the increase of $r / b$, i.e., larger radius of crease curvature, the so-called snap-through effect was weakened.

\subsection{Strip length to width ratio, $L / b$}

The influence of $L / b$ on the mechanical response of creased strips was investigated with its value ranging from 8 to 12 and the other parameters fixed at: $r=0.1 \mathrm{~mm}, \theta=2.09 \mathrm{rad}$, $t=0.1 \mathrm{~mm}$ (Fig. 6b). The results revealed that the smaller the value of $L / b$ was (shorter strip), the larger the peak moment would be. The peak moment decreased from around 725 to around $487 \mathrm{Nmm}$ with $L / b$ increased from 8 to 12. After the snap-through, all the obtained moment-rotation curves were almost identical, showing little influence of $L / b$ on the mechanical response of creased strips at this stage. Noticeably, $L / b$ had little influence on the snap-through itself, which was different from the influence of $r / b$. We also found that, after the snap-through, the localized fold could be regarded as an elastic hinge with small bending stiffness for all the creased strips, as the moment-rotational response in this range is linear.

\subsection{Initial opening angle, $\theta$}

The influence of the initial opening angle on the mechanical response of creased strips was studied when $\theta$ changed from 1.74 to $2.44 \mathrm{rad}$ and the other parameters were fixed at: $r=0.1 \mathrm{~mm}, L=100 \mathrm{~mm}, t=0.1 \mathrm{~mm}$ Fig. $6 c$ plotted the moment-rotation curves of the studied strips of different opening angles. It could be seen that the peak moment
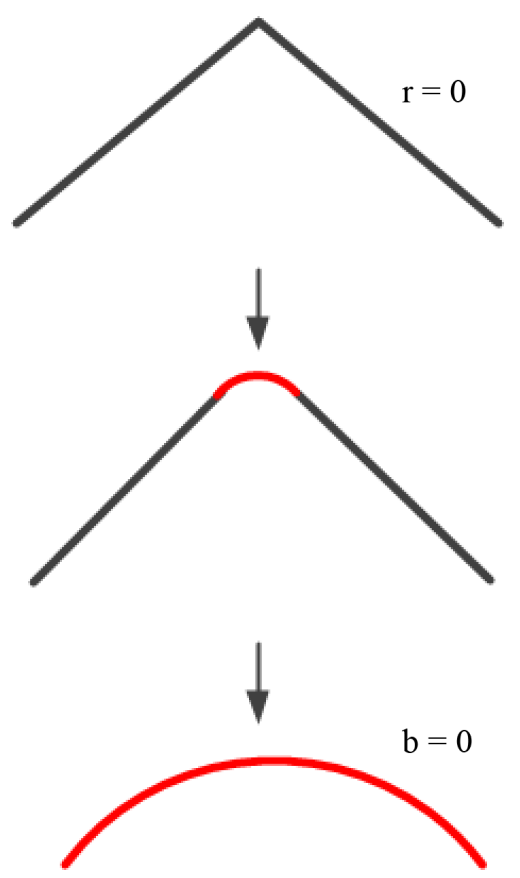

Figure 8. Schematic diagram of the transition from creased strip $(r=0)$ to curved strip $(b=0)$.

decreased from around 1161 round $487 \mathrm{~N} \mathrm{~mm}$ with increasing initial opening angle from 1.74 to $2.44 \mathrm{rad}$. The initial opening angle had a small influence on the snap-through effect. It should be noted that, when the initial opening angle increased to $2.44 \mathrm{rad}$, there was a sudden jump in the moment in the bending process (highlighted in Fig. $6 \mathrm{c}$ as dashed rectangular). The sudden change in moment was due to the transition of localized fold from single mode (one localized fold, Fig. 7a) to double mode (two localized folds, Fig. 7b), showing a bifurcate phenomenon. The region between the two localized folds was relatively flat.

\subsection{Strip thickness to width ratio, $t / b$}

The influence of $t / b$ on the mechanical response was also investigated when it varied from 0.01 to 0.05 and the other parameters were fixed at: $r=0.1 \mathrm{~mm}, L=100 \mathrm{~mm}, \theta=$ $2.09 \mathrm{rad}$. As shown in Fig. 6d, the strip thickness to width ratio had significant influence on the moment. The peak moment increased from around 570 to around $9815 \mathrm{~N} \mathrm{~mm}$ when $t / b$ increased from 0.01 to 0.05 , excessively larger than the moment values previously obtained. After snap-through, thicker strips showed much higher moments with no negative moment observed.

\subsection{Transition from a creased strip to a curved strip}

The difference between a curved strip and a creased strip lies in the different radius of curvature that links the two facets, as illustrated in Fig. 8. To further investigate the me- 


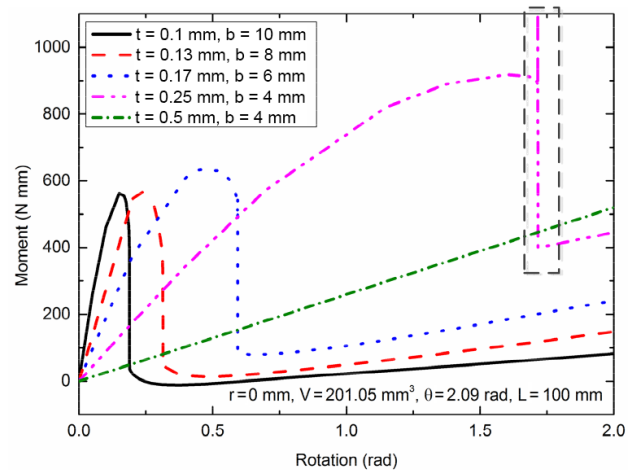

(a)

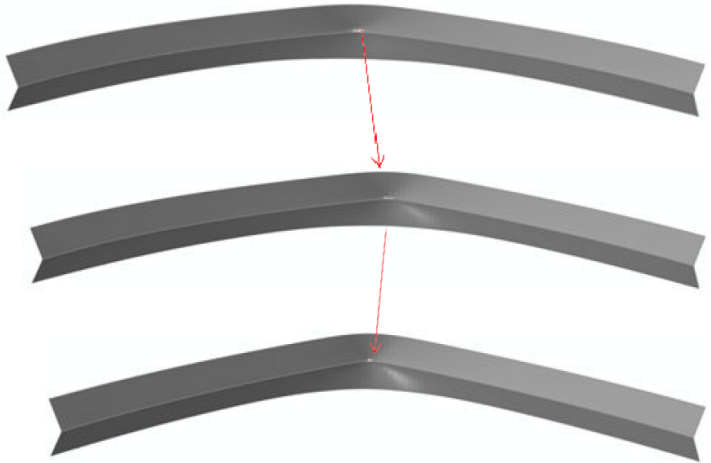

(b)

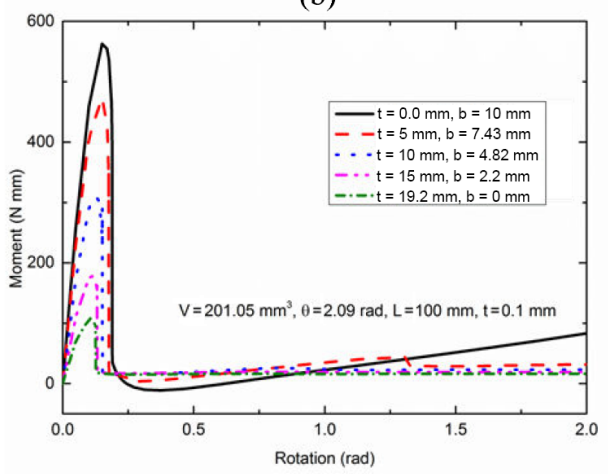

(d)

(c)

Figure 9. (a) Moment-rotation relationships obtained from finite element modelling showing the influence of the width of the rectangular facet $(b)$ and the thickness of strip $(t)$ on the mechanical response of the strip of $r=0$ when the volume $(V)$ and the length $(L)$ of the strip were constant, (b) the travelling of the localized fold during bending for the creased strip when $t=0.25 \mathrm{~mm}$ and $b=4 \mathrm{~mm}$, (c) deformed shape of the creased strip when $t=0.50 \mathrm{~mm}$ and $b=2 \mathrm{~mm}$, and (d) moment-rotation relationships obtained from finite element modelling showing the influence of the radius of the crease curvature $(r)$ and the width of the rectangular facet $(b)$ on the mechanical response with fixed values of the thickness of strip $(t)$, the initial opening angle $(\theta)$, the volume $(V)$, and the length $(L)$.

chanical response of strips during bending when the strip was transformed from creased-shape to curved-shape, we assumed that the mass/volume of the strip maintained unchanged. Therefore, the volume of the strip could be defined by

$$
V=[2 b+(\pi-\theta) r] t L
$$

Firstly, we studied the influence of the width of the rectangular facet $(b)$, and the uniform thickness of the strip $(t)$ on the overall mechanical behaviour of the strip of $r=0$ by keeping the volume $(V)$ and the length of the strip $(L)$ constant, i.e. $V=201.05 \mathrm{~mm}^{3}, L=100 \mathrm{~mm}$. Then we systematically investigated the transition from a creased strip to a curved strip by varying the radius of the crease curvature $(r)$ and the width of the rectangular facet $(b)$, with the fixed values of the initial opening angle $\theta$, and the uniform thickness of the strip $(t)$, the volume $(V)$ and the length of the strip $(L)$, e.g., $\theta=2.09 \mathrm{rad}, t=0.1 \mathrm{~mm}, V=201.05 \mathrm{~mm}^{3}, L=100 \mathrm{~mm}$.

\subsubsection{Strip with a line crease}

Line crease occurred when the radius of the crease curvature $(r)$ was zero (Fig. 8). Figure 9a compared the momentrotation curves of strips with different $b$ and $t$ values. The results showed that the moment-rotation curve shifted to the right when simultaneously increased the value of $t$ and decreased the value of $b$, with the peak moment increasing from around $563 \mathrm{~N} \mathrm{~mm}(t=0.1 \mathrm{~mm}, b=10 \mathrm{~mm})$ to around $1181 \mathrm{Nmm}(t=0.25 \mathrm{~mm}, b=4 \mathrm{~mm})$ in the studied range of rotation. For the ceased strip when $b=4 \mathrm{~mm}$ and $t=$ $0.25 \mathrm{~mm}$, a jump was observed before the formation of localized fold (highlighted in the dotted rectangular). The moment jumped to a very large value, and then plummeted to a relatively small value, which was caused by the movement of the localized fold during the bending process as demonstrated in Fig. 9b, indicating a metastable state. We could also find that a much larger amount of energy was needed to bend this creased strip (the area enclosed by the moment-rotation curve was much larger than those of other strips), which could be used as energy absorbing structures. For the strip 


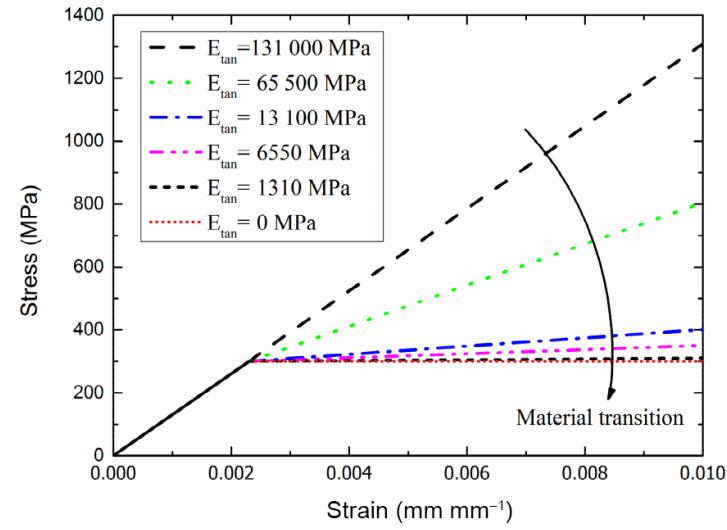

(a)

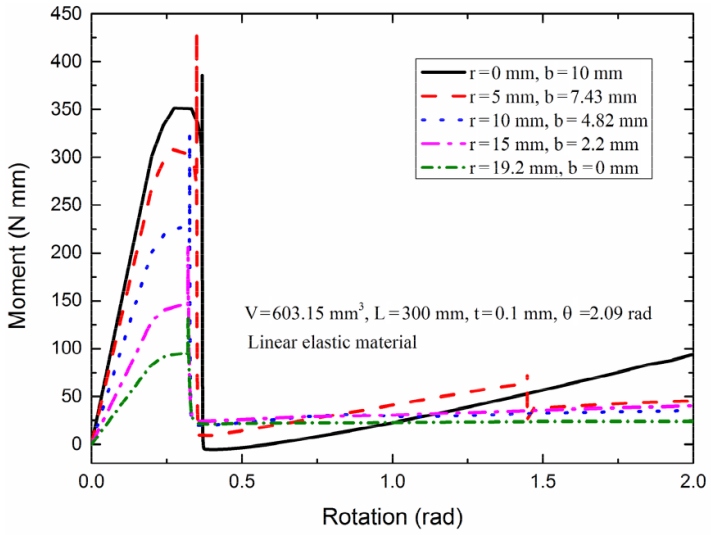

(b)

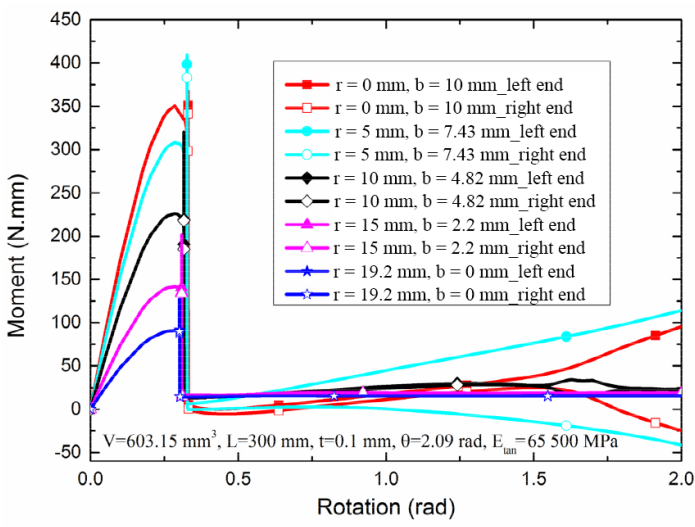

(c)

Figure 10. (a) Ideal true stress-strain curves of different materials with changing tangent moduli, (b) moment-rotation curves obtained from finite element modelling by using linear elastic material model, and (c) by using bilinear material model with the tangent modulus $E_{\tan }=65500 \mathrm{MPa}$.

with $t=0.5 \mathrm{~mm}$ and $b=2 \mathrm{~mm}$, no peak moment was found in the studied rotation range as shown in Fig. 9a. Figure 9c indicated that localized fold had not occurred for this strip in the studied range. These findings showed that the width of the rectangular facet and the thickness together significantly influenced the overall mechanical behaviour of the creased strip when the radius of the crease curvature was zero, i.e., line crease.

\subsubsection{Transition from a strip with a line crease to a curved strip}

Figure 9d showed the mechanical responses for various thin strips when they were transforming from line strip to a curved strip. The thickness $(t=0.1 \mathrm{~mm})$, the initial opening angle $(\theta=2.09 \mathrm{rad})$, the volume $\left(V=201.05 \mathrm{~mm}^{3}\right)$ and the length $(L=100 \mathrm{~mm})$ of the strip were fixed. The radius of crease curvature $(r)$ and the width of rectangular facet $(b)$ varied. The moment decreases from 562.89 to $107.97 \mathrm{~N} \mathrm{~mm}$, with the simultaneous increase of the radius of the crease curvature and decrease of the width of the rectangle facet.
We revealed that the moment of the strip varied significantly during the transition.

\section{Transition from a linear elastic material to an elastic-perfectly plastic material}

The application of origami always involves linear elastic materials in numerical analyses. However, it shows increasing applications in structural applications such as energy absorption structures, where material plasticity is often considered, e.g., aluminium or steel thin strips. Therefore, to reveal the influence of material properties on the mechanical response of creased strips, six types of materials considering elasticity and plasticity were applied as illustrated in Fig. 10a with different tangent modulus $\left(E_{\mathrm{tan}}\right)$. Other material coefficient included Young's modulus $E=131000 \mathrm{MPa}$, Poisson's ration $v=0.30$ and yield stress $\sigma_{\mathrm{ys}}=300 \mathrm{MPa}$. All the geometrical parameters were kept the same with those in Sect. 4.5.1 unless otherwise noted, which were determined by Eq. (2) with the same volume. Typically, $V=603.15 \mathrm{~mm}^{3}, L=300 \mathrm{~mm}$ $t=0.1 \mathrm{~mm}$, and $\theta=2.09 \mathrm{rad}$. A larger strip length $L$ was used to reduce the boundary effect. For each material, the 

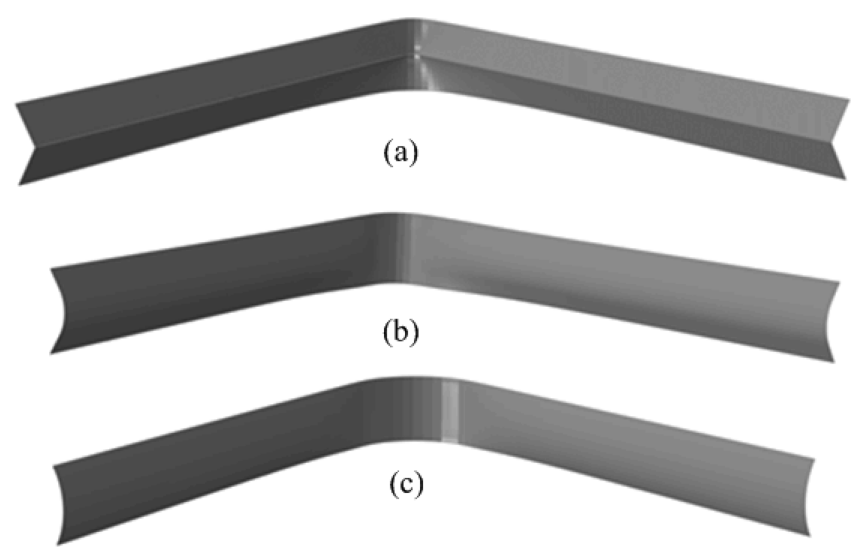

Figure 11. Typical deformed shapes of different strips in the finite element modellings by using bilinear material model with the tangent modulus $E_{\mathrm{tan}}=65500 \mathrm{MPa}$ : (a) strip with $r=0 \mathrm{~mm}$ and $b=10 \mathrm{~mm}$, (b) strip with $r=10 \mathrm{~mm}$ and $b=4.82 \mathrm{~mm}$, and (c) strip with $r=19.2 \mathrm{~mm}, b=0 \mathrm{~mm}$.

mechanical behaviour of the strip with various shapes from line to curved creases was extensively studied.

The moment-rotation curves for the linear elastic material, the bilinear material with the tangent modulus $\left(E_{\mathrm{tan}}\right)=$ $65500,13100,6550$, and $1310 \mathrm{MPa}$, and the elasticperfectively plastic material was plotted in Figs. 10b-c and $12 \mathrm{a}-\mathrm{d}$, respectively. For all the curves, the moment became smaller when simultaneously increased the value of $b$ and decreased the value of $r$ in the studied range of rotation, and the localised fold formed almost at the same rotational angle. Clear discrepancy was found at the stage after the formation of localised folds when the materials varying from elasticity to plasticity. Noticeably, the two ends of the strips behave differently for different materials. The moments of the two ends, i.e., left and right ends, of the thin strip behaved the same when linear elastic material was used. While, the moments of the two ends became evidently different when elasto-plastic materials were used, with the difference enlarged at smaller tangent modulus. It showed that the moment of the left end strip was increasing while it decreased for the right end strip because of the influence of asymmetrical boundary conditions in the finite element modelling and the plastic deformation in the localised fold region. Part of the moment was consumed to generate kinetic energy to produce plastic deformation when it was transited from left to right end when smaller tangent modulus was applied. When the tangent modulus became zero, i.e., elastic-perfectively plastic material, most of the moment was consumed, resulting in the largest difference of moment at the two ends of the strip. It was interesting to find that some curves, i.e. when $r=5 \mathrm{~mm}, b=7.43 \mathrm{~mm}$, the right end of the strip showed a decrease in moment after the snap-through as the rotation angle increased (see Fig. 10c), which might be due to the use of the non-linear material. It was also noteworthy that there were several jumps in the curves, which might be caused by the use of the non-linear material as well. Figure 11a$\mathrm{c}$ presented three typical deformed shapes for three thin strips ( $r=0 \mathrm{~mm}, b=10 \mathrm{~mm} ; r=10 \mathrm{~mm}, b=4.82 \mathrm{~mm}$; and $r=19.2 \mathrm{~mm}, b=0 \mathrm{~mm})$ when the tangent modulus $\left(E_{\tan }\right)=$ $65500 \mathrm{MPa}$, which showed that the localised fold region became larger and more flat as simultaneously decreased the value of $b$ and increased the value of $r$.

\section{Discussion}

The introduction of a simple crease to a thin strip was numerically studied to reveal the influence of the crease on the overall mechanical behaviour of the strip. Compared with curved and flat strips, the creased strip exhibited remarkably different mechanical behaviour. When a crease was introduced to a flat strip, we observed the magnitude of the peak moment increased by 100 times, demonstrating the dramatic influence of the simple crease on the mechanical behaviour of the strip.

As revealed in the parametric studies, the moment-rotation curves of bending the creased strips showed typical three distinct stages. Firstly, the moment increased to the peak value at a small rotation angle, then it dropped freely to show a snap-through procedure due to the formation of localized folds, which was followed by a final stage of smooth increasing in the moment to a stable value. The geometry of the creased strip could tremendously influence its mechanical response. The dimensionless parameters $t / b$ and $r / b$ were found to have the greatest and the least influence on the mechanical response of the creased strips, respectively. We also found that the moment-rotation curves were very similar to each other after the formation of localized folds for strips with various values of $L / b$, and $\theta$. Furthermore, the localized fold could trigger two different folds on the same strip if the initial opening angle was sufficiently large, e.g., $2.44 \mathrm{rad}$ (Fig. 7). When the strip transformed from line-creased structure to curved structure, the change in geometrical parameters $r$ and $b$ significantly influenced the moment of the strip. It should be noted that not all the strips formed the localized folds. For example, the creased strip with $t=0.50 \mathrm{~mm}$ and $b=2 \mathrm{~mm}$ (Fig. 9c) had no localized fold, which is similar to the flat strip as shown in Fig. 3a.

The investigation into the influence of the transition of the strip material from a linear elastic material to an elasticperfect plastic material showed that material properties had significant influence on the overall behaviour of the creased strip. The moments of the two ends of the creased strip were the same when a linear elastic material was used since the transmission of the moment was not interfered by the elastic fold. However, the increasingly localized plastic deformation in the elasto-plastic material affected the transmission of the moments through the plastic folds during the bending, which became more evident when the tangent modulus was smaller. 


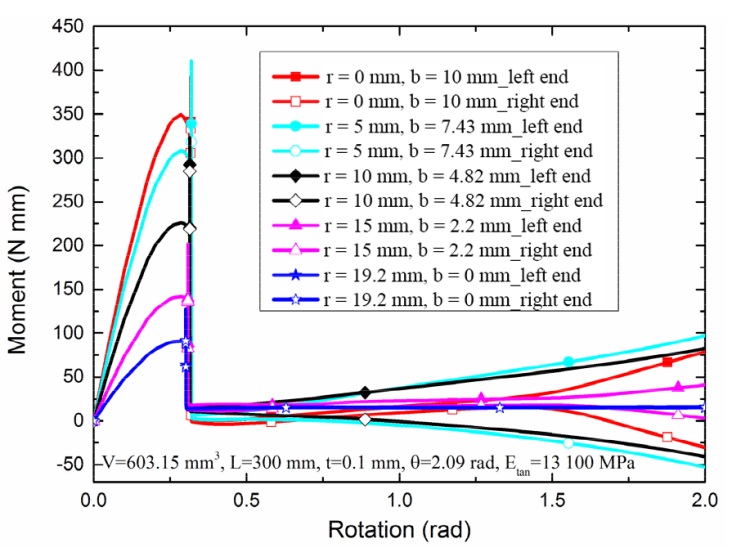

(a)

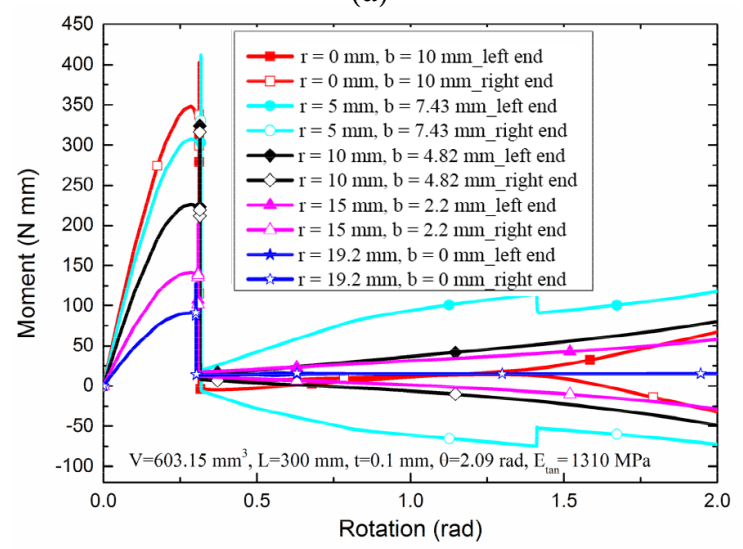

(c)

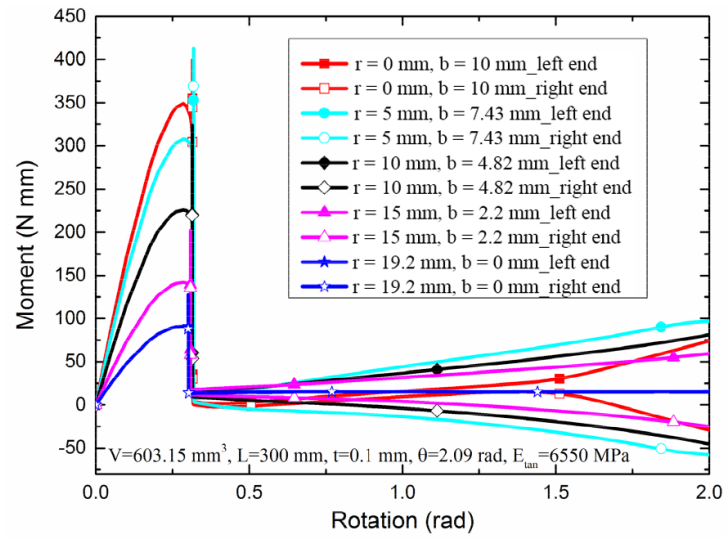

(b)

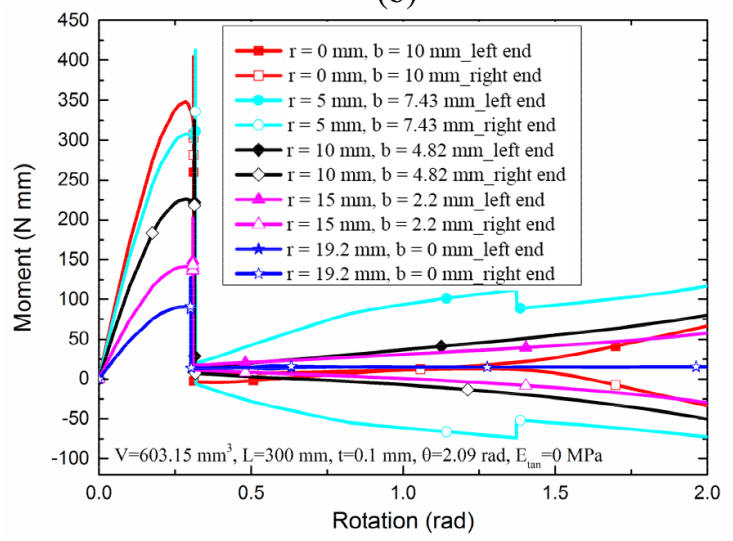

(d)

Figure 12. Moment-rotation curves obtained from finite element modelling by using bilinear material model with different tangent modulus (a) $E_{\tan }=13100 \mathrm{MPa}$, (b) $E_{\tan }=6550 \mathrm{MPa}$, (c) $E_{\tan }=1310 \mathrm{MPa}$, and (d) $E_{\tan }=0 \mathrm{MPa}$ (elastic-perfectly plastic material).

\section{Conclusions}

In this paper the mechanical behaviour of a creased thin strip under opposite-sense bending was studied via finite element modelling which was verified by physical models. The effects of the dimensionless parameters, including $r / b$, $L / b, \theta$, and $t / b$, on the overall mechanical behaviour of the creased strip were systematically investigated. The results revealed that the introduction of a simple crease to the thin strip could significantly alter the flexural performance of the strip. The uniform thickness and the radius of the creased segment had the greatest and the least influence on the mechanical behaviour of the creased strip, respectively. The investigation of the transition from a creased strip to a curved strip uncovered the tremendous influence of the geometrical parameters on the overall mechanical behaviour of the strip. We also showed that the material properties could play a key role in the bending of the creased strip. Furthermore, deformed shapes of the creased strips from the finite element simulations agreed well with those of the physical models made from thin metal strips. The findings from this study provide useful information for designing origami struc- tures using creased thin strips. Specifically, they can guide engineers to design self-folding structures, energy absorbing structures and others.

Data availability. The data underlying this article are not available by agreement with our partners to protect their commercial confidentiality.

Competing interests. The authors declare that they have no conflict of interest.

Acknowledgements. The first author would like to thank the China Scholarship Council for scholarship (201506130053). This research was also financially supported by the Australian Research Council through a Discovery Project grant (DP160101400) and the State Key Laboratory of Advanced Design and Manufacturing for Vehicle Body at Hunan University through an open fund (31615003). 
Edited by: Guangbo Hao

Reviewed by: Shuang $\mathrm{Xu}$ and one anonymous referee

\section{References}

Chen, Y., Peng, R., and You, Z.: Origami of thick panels, Science, 349, 396-400, 2015.

Couturier, E., Dumais, J., Cerda, E., and Katifori, E.: Folding of an opened spherical shell, Soft Matter, 9, 8359-8367, 2013.

Delimont, I. L., Magleby, S. P., and Howell, L. L.: Evaluating compliant hinge geometries for origami-inspired mechanisms, J. Mech. Robot., 7, 011009, https://doi.org/10.1115/1.4029325, 2015.

Dias, M. A. and Audoly, B.: A non-linear rod model for folded elastic strips, J. Mech. Phys. Solids, 62, 57-80, 2014.

Dias, M. A., Dudte, L. H., Mahadevan, L., and Santangelo, C. D.: Geometric mechanics of curved crease origami, Phys. Rev. Lett., 109, 114301, https://doi.org/10.1103/PhysRevLett.109.114301, 2012.

Filipov, E. T., Tachi, T., and Paulino, G. H.: Origami tubes assembled into stiff, yet reconfigurable structures and metamaterials, $\mathrm{P}$. Natl. Acad. Sci. USA, 112, 12321-12326, 2015.

Francis, K. C., Blanch, J. E., Magleby, S. P., and Howell, L. L.: Origami-like creases in sheet materials for compliant mechanism design, Mech. Sci., 4, 371-380, 2013.

Gething, M. J. and Sambrook, J.: Protein folding in the cell, Nature, 355, 33-45, 1992.

Hanna, B. H., Lund, J. M., Lang, R. J., Magleby, S. P., and Howell, L. L.: Waterbomb base: a symmetric single-vertex bistable origami mechanism, Smart Mater. Struct., 23, 094009, https://doi.org/10.1088/0964-1726/23/9/094009, 2014.

Jayaram, K. and Full, R. J.: Cockroaches traverse crevices, crawl rapidly in confined spaces, and inspire a soft, legged robot, $\mathrm{P}$. Natl. Acad. Sci. USA, 113, E950-E957, 2016.

Lechenault, F., Thiria, B., and Bedia, M. A.: Mechanical response of a creased sheet, Phys. Rev. Lett., 112, 244301, https://doi.org/10.1103/PhysRevLett.112.244301, 2014.

Lin, S., Xie, Y. M., Li, Q., Huang, X., and Zhou, S.: A kirigami approach to forming a synthetic buckliball, Sci. Rep., 6, 33016, https://doi.org/10.1038/srep33016, 2016.

Na, J. H., Evans, A. A., Bae, J., Chiappelli, M. C., Santangelo, C. D., Lang, R. J., Thomas, C. H., and Hayward, R. C.: Programming reversibly self-folding origami with micropatterned photocrosslinkable polymer trilayers, Adv. Mater., 27, 79-85, 2015.
Overvelde, J. T., Weaver, J. C., Hoberman, C., and Bertoldi, K.: Rational design of reconfigurable prismatic architected materials, Nature, 541, 347-352, 2017.

Schenk, M. and Guest, S. D.: Origami folding: A structural engineering approach, In Origami 5: Fifth International Meeting of Origami Science, Mathematics, and Education CRC Press, Boca Raton, FL, 291-304, 2011.

Soykasap Ö.: Analysis of tape spring hinges, Int. J. Mech. Sci., 49, 853-860, 2007.

Seffen, K. A. and Pellegrino, S.: Deployment dynamics of tape springs, Proc. R. Soc. Lon. Ser.-A, 455, 1003-1048, 1999.

Seffen, K. A., You, Z., and Pellegrino, S.: Folding and deployment of curved tape springs, Int. J. Mech. Sci., 42, 2055-2073, 2000.

Walker, M. G. and Seffen, K. A.: Flexural mechanics of creases in thin metallic strips, Proceedings of the IASS Annual Symposium, Spatial Structures in the 21st Century, 26-30 September 2016, Tokyo, Japan, 2016.

Walker, S. J. I. and Aglietti, G. S.: Experimental investigation of tape springs folded in three dimensions, AIAA J., 44, 151-159, 2006.

Yang, H., Liu, R., Wang, Y., Deng, Z., and Guo, H.: Experiment and multiobjective optimization design of tape-spring hinges, Struct. Multidiscip. O., 51, 1373-1384, 2015.

Yang, K., Xu, S., Shen, J., Zhou, S., and Xie, Y. M.: Energy absorption of thin-walled tubes with pre-folded origami patterns: numerical simulation and experimental verification, Thin Wall. Struct., 103, 33-44, 2016.

Yang, K., Xu, S., Shen, J., Zhou, S., and Xie, Y. M.: Design of dimpled tubular structures for energy absorption, Thin Wall. Struct., 112, 31-40, 2017

Yasuda, H. and Yang, J.: Reentrant origami-based metamaterials with negative Poisson's ratio and bistability, Phys. Rev. Lett., 114, 185502, https://doi.org/10.1103/PhysRevLett.114.185502, 2015.

Ye, H., Zhang, Y., Yang, Q., Xiao, Y., Grandhi, R. V., and Fischer, C. C.: Optimal design of a three tape-spring hinge deployable space structure using an experimentally validated physics-based model, Struct. Multidiscip. O., 56, 973-989, 2017. 